\title{
Segment 6 monosegment-preserving hepatectomy for hepatoblastoma: individualizing treatment beyond the resectability criteria
}

\author{
Chee-Chien Yong, Chao-Long Chen, Zhihao Li, Aldwin D. Ong \\ Liver Transplantation Center, Department of Surgery, Kaohsiung Chang Gung Memorial Hospital, Kaohsiung \\ Correspondence to: Chao-Long Chen, MD, PhD (Hon). Liver Transplantation Center, Department of Surgery, Kaohsiung Chang Gung Memorial \\ Hospital, 123 Ta-Pei Road, Niao-Sung, Kaohsiung. Email: clchen@cgmh.org.tw.
}

Submitted Apr 29, 2020. Accepted for publication Jul 24, 2020.

doi: $10.21037 / \mathrm{hbsn}-20-457$

View this article at: http://dx.doi.org/10.21037/hbsn-20-457

Monosegment-preserving hepatic resections are very rarely considered in patients with locally advanced tumors given the high risk of liver failure from an inadequate liver remnant $(1,2)$. Where this is considered, the associating liver partition and portal vein ligation for staged hepatectomy (ALPPS) technique, a two-staged procedure in order to induce remnant volume hypertrophy, have been reported $(1,2)$. However, ALPPS has been known to have a high complication rate, hence even more infrequently, this complicated technique has been contemplated for pediatric patients (2). In the case we present, we identified an infant with a locally advanced hepatoblastoma with a unique anatomic presentation, that certainly precludes the possibility of ALPPS, but which otherwise provided the team with the opportunity to perform a single-staged monosegment-preserving hepatectomy with curative intent, in lieu of the alternative of liver transplantation (LT).

A 9-month old girl was referred to our institution for evaluation of a large liver mass discovered on ultrasound that was assessed as the probable cause of recurrent fever, poor intake and abdominal distention. On abdominal CT, a heterogeneously enhancing, unifocal liver mass $(14 \mathrm{~cm} \times$ $11 \mathrm{~cm} \times 9 \mathrm{~cm}$ ) was detected involving segments $3 / 4 / 5 / 7 / 8$, all three hepatic veins (LHV, MHV and RHV), and both portal vein branches (LPV and RPV) (Figures 1A,B,C). Her alphafetoprotein (AFP) was markedly elevated at $470 \times 10^{6} \mathrm{ng} / \mathrm{mL}$. Biopsy confirmed our diagnostic impression of hepatoblastoma, pre-treatment extent of disease (PRETEXT) stage IV.

The patient received neoadjuvant chemotherapy with cisplatin and epirubicin but developed heart failure after the first cycle (left ventricular dilatation and elevated brain natriuretic peptide). Epirubicin was discontinued and replaced with carboplatin and etoposide for another two cycles concurrent with cisplatin. The disease had a corresponding partial response, with the tumor regressing from $14 \mathrm{~cm} \times 11 \mathrm{~cm}$ to $10 \mathrm{~cm} \times 7 \mathrm{~cm}$, and AFP decreasing to $3,416 \mathrm{ng} / \mathrm{mL}$. No regional or distant metastases were detected on whole body FDG-PET scan. While the LHV, MHV, RHV, and RAPV remained involved by the tumor, clear margins appeared achievable given the CT and Doppler ultrasound-assessed adequate inflow and outflow via the RPPV and consequently tumor-free IRHV respectively (Figure 2A,B,C).

When the patient was re-evaluated for surgery after neoadjuvant treatment, her body weight was noted at $9.5 \mathrm{~kg}$. The calculated remnant liver volume (RLV) to body weight ratio (BWR) was $1.8 \%(169 \mathrm{~mL} / 9.5 \mathrm{~kg})$. Upon explaining that this was adequate RLV for the infant, with a contingency for bail-out and elective living donor liver transplantation (LDLT) if intraoperatively deemed unresectable, the parents consented to the surgery. An extended left trisegmentectomy including segment 1 was subsequently performed preserving only the segment 6 (S6), its S6 Glissonian pedicle, and the IRHV (Figure 3A,B). Gross pathology revealed a well-defined, $9 \mathrm{~cm} \times 8 \mathrm{~cm} \times$ $4 \mathrm{~cm}$ large tumor with components of calcified necrosis and a resection margin of $2 \mathrm{~mm}$. Microscopically, the tumor cells were of mixed epithelial-mesenchymal type. The patient recovered well without developing signs of liver failure. On follow-up CT one month after resection, the remnant liver showed parenchymal regeneration to a volume of $323 \mathrm{~mL}$ 

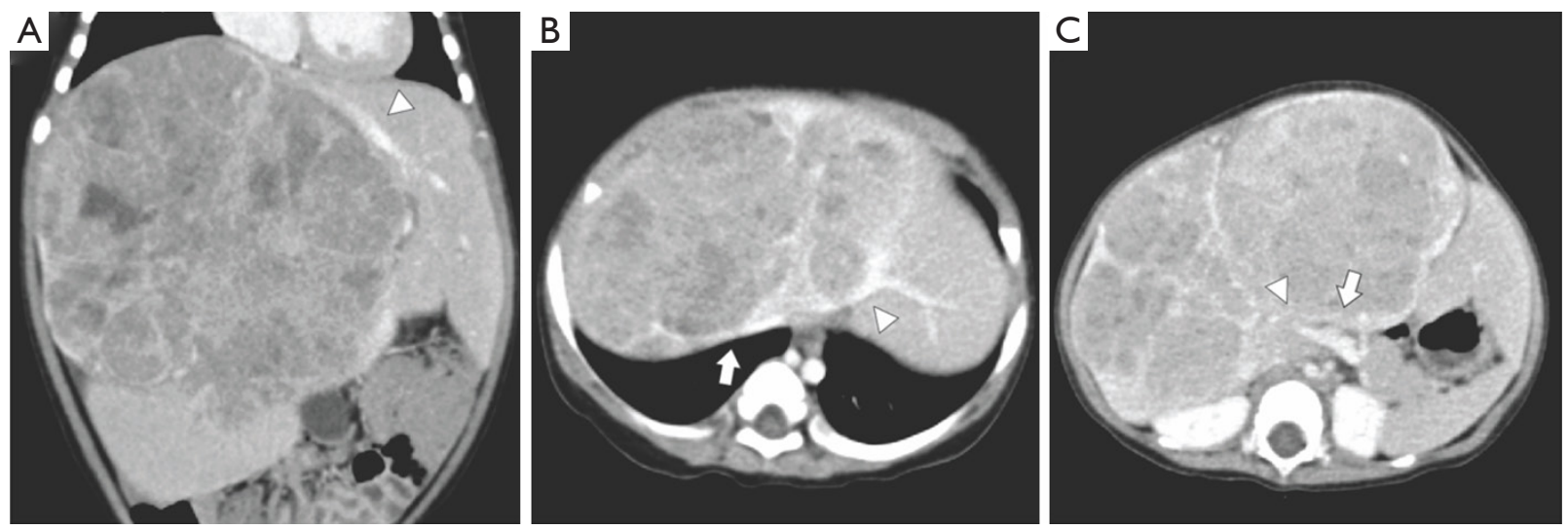

Figure 1 Initial presentation of the hepatoblastoma $(14 \mathrm{~cm} \times 11 \mathrm{~cm} \times 9 \mathrm{~cm})$ by CT angiography. Tumor shows involvement of the (A) MHV (arrowhead), (B) LHV (arrowhead), RHV (arrow), (C) LPV (arrow) and RPV (arrowhead). MHV, middle hepatic vein; LHV, left hepatic vein; RHV, right hepatic vein; LPV, left portal vein; RPV, right portal vein.
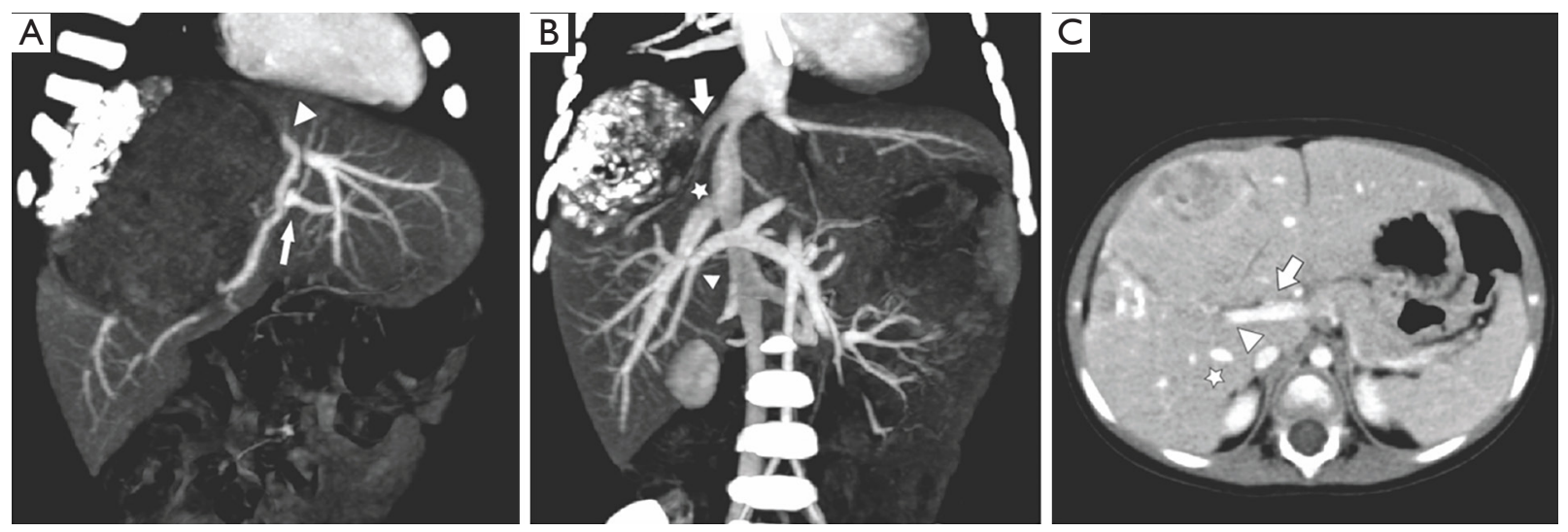

Figure 2 Follow-up CT angiography after neoadjuvant chemotherapy. Persistent tumor involvement seen in (A) LHV-MHV (arrowhead), LPV (arrow), and (B) RHV (arrow), with IRHV (star) and RPPV (arrowhead) noted to be free of tumor. (C) LPV (arrow) seen encased by the tumor, while the RPPV (arrowhead) and the IRHV (star) were tumor-free. LHV, left hepatic vein; MHV, middle hepatic vein; LPV, left portal vein; RHV, right hepatic vein; IRHV, inferior right hepatic vein; RPPV, right posterior portal vein.

$(\mathrm{RLV} / \mathrm{BWR}=3.4 \%$ ) (Figure $3 \mathrm{C}$ ) while AFP normalized to $8 \mathrm{ng} / \mathrm{mL}$. The patient was discharged well.

Hepatoblastoma is the most common primary pediatric liver tumor with an annual incidence of $1.2-1.5$ per million (3). Over the last four decades, the survival outcome of hepatoblastoma has improved from $30 \%$ to $80 \%$ due largely to improvements in chemotherapy protocols and liver resection (LR) techniques (3). Furthermore, current management is based on risk stratification developed by multicenter trial groups, thereby improving stage-specific management and survival outcomes (4).

The PRETEXT staging system, consisting of the group and the annotation factors, is the most critical risk stratification tool for hepatoblastoma. The PRETEXT group (I-IV) describes the extension of tumor within the liver and is determined by the number of contiguous tumor-free liver sections. The annotations characterize features associated with tumor extension beyond the liver parenchyma, including hepatic vein/portal vein involvement $(\mathrm{V} / \mathrm{P})$, extrahepatic disease $(\mathrm{E})$, tumor rupture $(\mathrm{R})$, multifocality $(\mathrm{F})$, and metastatic disease (to both lungs and lymph nodes) (M) (5).

On presentation, the patient was staged PREXTEXT IV (no tumor-free section), M-, P+ (involvement of both portal vein branches), $\mathrm{V}+$ (involvement of all three hepatic veins), ERF- (5). She had an unresectable tumor classified 

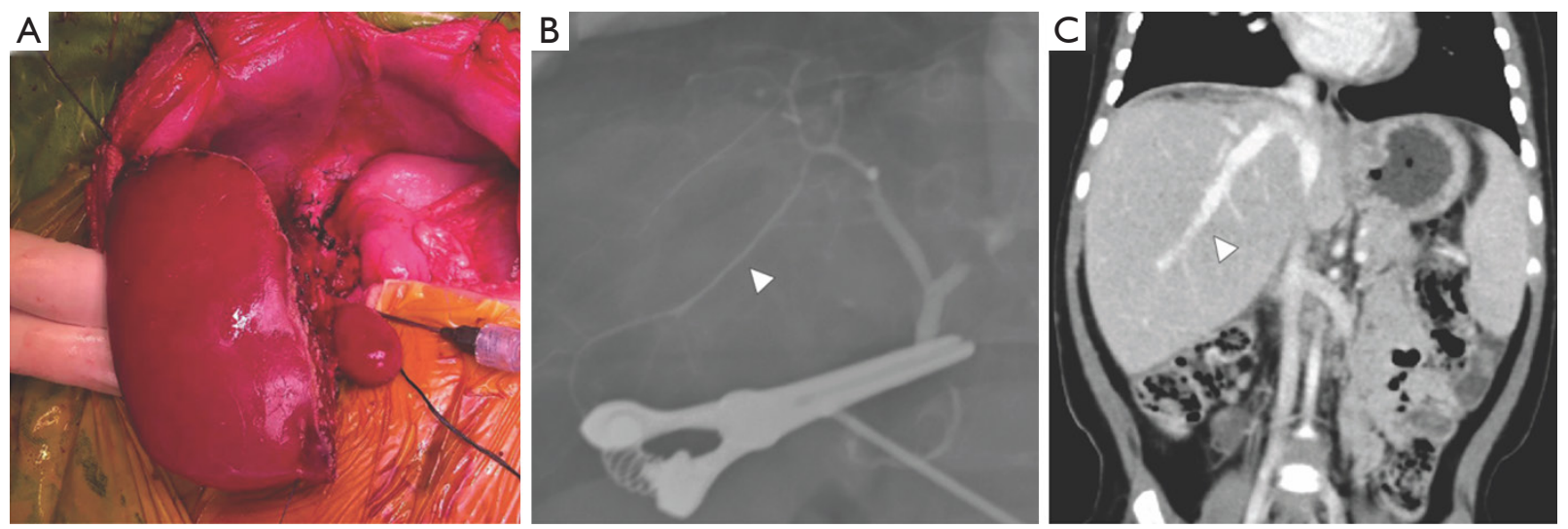

Figure 3 Intraoperative images and postoperative follow-up CT angiogram. (A) Remnant S6 monosegment; (B) intraoperative cholangiography illustrating patent S6 bile duct (arrowhead); (C) one-month post-resection CT presenting a well-regenerated liver with no signs of recurrence and a patent IRHV (arrowhead). IRHV, inferior right hepatic vein.

as high risk, and requiring neoadjuvant chemotherapy (6). Cisplatin and doxorubicin are the two common first-line agents recommended by the study groups. In consideration of doxorubicin-induced cardiotoxicity, epirubicin, which has a lower toxicity profile, was used. Unfortunately, the patient still developed clinical cardiotoxicity, which prompted the early discontinuation of epirubicin, and potentially resulting in a suboptimal neoadjuvant response. While the RPPV became tumor-free $(\mathrm{P}-)$, the tumor remained POSTTEXT stage IV (V+, ERF-).

Following the traditional paradigm of liver resectability which is defined as the removal of tumor with negative margins, and preservation of $\geq 2$ contiguous liver segments along with their inflow, outflow and biliary drainage, the tumor would be classified as unresectable. With our institution's extensive experience in LDLT, the patient would technically be considered as a suitable candidate for LT, with favorable expected outcome (6). Nevertheless, with consideration of several key elements specific to the infant, we favored LR over LT.

This pediatric patient had the anatomic privilege of possessing a sizeable IRHV that was tumor-free after chemotherapy. With RPPV and IRHV as inflow and outflow respectively, the tumor-free S6 could hypothetically be preserved following resection. Moreover, while monosegment-preservation is frequently insufficient to support physiologic liver function, it was deemed that the patient would have an adequate RLV after singlestage oncologic resection, given the already notable compensatory hypertrophy of the liver remnant, which was surmised as being due to tumor compression and functional loss of the affected liver segments. It was deliberated that ALPPS procedure, with its known high morbidity, would be both risky and redundant for the patient $(1,2)$. To our knowledge, this unique pathoanatomic constellation and compensatory sequelae that allowed us to perform a singlestage S6 monosegment-preserving hepatectomy, has not been previously reported.

Notwithstanding, this operation was perhaps more complex compared to LT since the inflow and outflow of the remnant liver must be preserved without compromise to the margin. The advantage on the other hand was that no vascular or biliary reconstruction was necessary, and on the background of a post-neoadjuvant R0-resection margin, survival outcome can be expected to be as favorable as patients undergoing LT for advanced disease $(7,8)$.

That being said, advanced hepatoblastoma should ideally be evaluated and treated in centers with expertise in complex LR because salvage LT is in itself not a safety net for recurrent disease. Patients undergoing a rescue LT after a failed LR have a significantly lower survival than primary LT $30 \%$ vs. 80\%) (9). However, for the patient, LR was decided over primary LT for two main reasons-firstly, the patient would be spared from life-long immunosuppression, and secondly, there was sufficient confidence that a comparably favorable survival outcome could be achieved with LR as with LT.

PRETEXT/POSTTEXT staging is a valuable tool in the risk stratification of hepatoblastoma and offers assistance in the initial evaluation for resectability. However, it fails to consider the relevance of accessory hepatic veins in altering possible surgical management. In the case presented, the authors understood this gap in the detail, 
before deliberating and successfully performing the first single-stage S6 monosegment-preserving hepatectomy for a liver malignancy in an infant. It is however not the authors' intention to propagate this technique as a standard procedure. Nevertheless, this case is a befitting illustration that the limit of surgical resectability can potentially be challenged by those who have a keen eye and intent for detail, and an extensive institutional experience on the surgical management of locally advanced hepatic malignancy.

\section{Acknowledgments}

Funding: None.

\section{Footnote}

Provenance and Peer Review: This article was a standard submission to the journal Hepatobiliary Surgery and Nutrition. The article has undergone external peer review.

Conflicts of Interest: All authors have completed the ICMJE uniform disclosure form (available at https://hbsn. amegroups.com/article/view/10.21037/hbsn-20-457/coif). Dr. CLC serves as an unpaid editorial board member of Hepatobiliary Surgery and Nutrition. The other authors have no conflicts of interest to declare.

Ethical Statement: The authors are accountable for all aspects of the work in ensuring that questions related to the accuracy or integrity of any part of the work are appropriately investigated and resolved. Written informed consent was obtained from the patient for publication of this manuscript and any accompanying images.

Open Access Statement: This is an Open Access article distributed in accordance with the Creative Commons Attribution-NonCommercial-NoDerivs 4.0 International License (CC BY-NC-ND 4.0), which permits the noncommercial replication and distribution of the article with the strict proviso that no changes or edits are made and the original work is properly cited (including links to both the formal publication through the relevant DOI and the license). See: https://creativecommons.org/licenses/by-nc-nd/4.0/.

\section{References}

1. Pineda-Solís K, Paskar D, Tun-Abraham M, et al.
Expanding the limits of resectability: Associating liver partition and portal vein ligation for staged hepatectomy (ALPPS) using monosegment 6, facilitated by an inferior right hepatic vein. J Surg Oncol 2017;115:959-62.

2. Wiederkehr JC, Avilla SG, Mattos E, et al. Associating liver partition with portal vein ligation and staged hepatectomy (ALPPS) for the treatment of liver tumors in children. J Pediatr Surg 2015;50:1227-31.

3. Czauderna P, Lopez-Terrada D, Hiyama E, et al. Hepatoblastoma state of the art: pathology, genetics, risk stratification, and chemotherapy. Curr Opin Pediatr 2014;26:19-28.

4. Czauderna P, Haeberle B, Hiyama E, et al. The Children's Hepatic tumors International Collaboration (CHIC):

Novel global rare tumor database yields new prognostic factors in hepatoblastoma and becomes a research model. Eur J Cancer 2016;52:92-101.

5. Towbin AJ, Meyers RL, Woodley H, et al. 2017 PRETEXT: radiologic staging system for primary hepatic malignancies of childhood revised for the Paediatric Hepatic International Tumour Trial (PHITT). Pediatr Radiol 2018;48:536-54.

6. Meyers RL, Tiao G, de Ville de Goyet J, et al. Hepatoblastoma state of the art: pre-treatment extent of disease, surgical resection guidelines and the role of liver transplantation. Curr Opin Pediatr 2014;26:29-36.

7. Feng J, He Y, Wei L, et al. Assessment of Survival of Pediatric Patients With Hepatoblastoma Who Received Chemotherapy Following Liver Transplant or Liver Resection. JAMA Netw Open 2019;2:e1912676.

8. McAteer JP, Goldin AB, Healey PJ, et al. Surgical treatment of primary liver tumors in children: outcomes analysis of resection and transplantation in the SEER database. Pediatr Transplant 2013;17:744-50.

9. Otte JB, Pritchard J, Aronson DC, et al. Liver transplantation for hepatoblastoma: results from the International Society of Pediatric Oncology (SIOP) study SIOPEL-1 and review of the world experience. Pediatr Blood Cancer 2004;42:74-83.

Cite this article as: Yong CC, Chen CL, Li Z, Ong AD. Segment 6 monosegment-preserving hepatectomy for hepatoblastoma: individualizing treatment beyond the resectability criteria. HepatoBiliary Surg Nutr 2021;10(1):142145. doi: 10.21037/hbsn-20-457 